\title{
Destabilization of Coxsackievirus B3 Genome Integrated with Enhanced Green Fluorescent Protein Gene
}

\author{
Lei Tong $^{\mathrm{a}}$ Lexun Lin ${ }^{\mathrm{a}}$ Wenran Zhao ${ }^{\mathrm{b}}$ Bo Wang ${ }^{\mathrm{a}}$ Shuaiqin $\mathrm{Wu}^{\mathrm{a}}$ \\ Huimin Liu ${ }^{\mathrm{a}}$ Xiaoyan Zhong ${ }^{\mathrm{a}}$ Yuqiong Cui ${ }^{\mathrm{a}}$ Hongxia Gu${ }^{\mathrm{a}}$ Fengmin Zhang ${ }^{\mathrm{a}}$ \\ Zhaohua Zhong a \\ Departments of a Microbiology and ${ }^{b}$ Cell Biology, Harbin Medical University, Harbin, China
}

\author{
Key Words \\ Coxsackievirus B3 - Enhanced green fluorescent protein • \\ Genome destabilization
}

\begin{abstract}
Aims: To evaluate the stability of coxsackievirus B (CVB) genome integrated with the enhanced green fluorescent protein gene (egfp) and provide valuable information for the use of the recombinant CVB variant. Methods: A CVB3 variant expressing eGFP was constructed by insertion of the egfp open-reading frame (ORF) at the $5^{\prime}$ end of CVB3 ORF. The recombinant virus CVB3-eGFP was serially passaged in HeLa cells. The deletions in the CVB3-eGFP genome around egfp were examined by reverse transcription polymerase chain reaction and sequencing. Results: Genomic deletions of CVB3-eGFP could be observed as early as the 2nd passage. Sequencing showed that the genomic deletions caused either viral ORF shifts or partial deletions of the viral VP4 coding sequence. The 6th passage of CVB3-eGFP was checked by plaque assay for eGFP expression. All plaque-like foci showed eGFP expression. eGFP expression was also viewed in HeLa cells infected with plaque-forming viruses. Conclusions: The insertion of egfp destabilized the CVB3 genome.
\end{abstract}

The genomic deletions led to lethal mutations because of the termination of viral protein synthesis due to viral ORF shift and loss of partial viral gene. These findings imply that experimental data based on CVB integrated with the reporter gene should be interpreted with caution.

Copyright $\odot 2011$ S. Karger AG, Basel

\section{Introduction}

Coxsackieviruses are members of the Picornaviridae family with a single-stranded RNA genome. These positive-sense viral RNA, about 7,400 nucleotides (nt) in length, are similar to messenger RNA (mRNA) and, thus, can be translated by the host cells [1]. Unlike cellular mRNA, the genome of coxsackievirus possesses an unusually long 5' untranslated region (5' UTR, approx. 740 nt) where an internal ribosomal entry site lies. The importance of the internal ribosomal entry site is to bind ribosomes and initiate viral protein synthesis without poly(A)-binding protein dependence $[2,3]$. In the entire genome of coxsackievirus, there is only 1 open reading frame (ORF) encoding a polyprotein precursor, which is cleaved by virus-encoded proteinases $2 \mathrm{~A}^{\text {pro }}$ and $3 \mathrm{C}^{\text {pro }}$

\section{KARGER}

Fax +4161306 1234

E-Mail karger@karger.ch

www.karger.com
() 2011 S. Karger AG, Basel

0300-5526/11/0545-0268\$38.00/0

Accessible online at:

www.karger.com/int
Zhaohua Zhong, PhD

Department of Microbiology, Harbin Medical University

Harbin, Heilongjiang Province 150081 (China)

Tel./Fax +86 45186685122

E-Mail zhongzh@ems.hrbmu.edu.cn 
into the mature structural and functional viral proteins [1]. Group B coxsackieviruses (CVB) are established important pathogens of human myocarditis, and are highly associated with the pathogenesis of dilated cardiomyopathy [4-7]. In spite of the critical role of CVB in myocarditis and cardiomyopathy, so far, the precise mechanism of CVB-related heart diseases has not been well defined.

Reporter genes were employed to detect virus replication and transcription quantitatively in a great number of studies [8-10]. Green fluorescent protein (GFP) of Aequorea victoria is one of the widely used reporters for the evaluation of gene expression and modulation $[11,12]$. It has been shown previously $[13,14]$ that CVB infection could be readily determined by fusing the ORF of the GFP gene $(g f p)$ to the $\mathrm{N}$ terminus of the CVB polyprotein. Upon pMKS-1, a plasmid carrying the full-length genome of the CVB3 H3 strain [15] with an engineered 3C protease cleavage site at the $5^{\prime}$ end of the coding region of CVB3 [16], a CVB3 variant integrated with the ORF of the enhanced GFP gene (egfp), was constructed in this study. Green fluorescence could be viewed in the cells infected with the recombinant virus, CVB3-eGFP, and viral biosynthesis could be easily monitored. However, we found that the insertion of egfp ORF caused destabilization of the CVB3 genome. CVB3-eGFP tended to delete the egfp ORF upon replications. A similar observation was also obtained from the study of poliovirus [17].

Furthermore, according to the study of poliovirus integrated with the $g f p$ gene [17], genomic deletions also produced some stable poliovirus variants that carried small leader polypeptides. Reverse transcription polymerase chain reaction (RT-PCR) and sequence analysis of the genomic RNAs reproducibly revealed a few preferred genotypes among the isolated deletion variants. This result suggests that the genomic deletions may cause a variant mixture (quasispecies) during the passage of the recombinant viruses in permissive cells. The presence of quasispecies without GFP expression could misguide the interpretation of studies on viral gene expression and modulation by using such a poliovirus variant.

So far, there is no detailed study available on the genomic stability of coxsackievirus integrated with the reporter gene. Though both CVBs and polioviruses belong to Picornaviridae, there are huge differences among the genomic sequences of CVBs and polioviruses. CVBs also differ from polioviruses in terms of tissue tropism and pathogenesis. To guide the use of reporter-integrated CVB variants for pathogenesis studies, the destabilization of the CVB3-eGFP genome was analyzed in this study.

Destabilization of CVB3 Genome Fused with $\operatorname{egfp}$

\section{Materials and Methods}

\section{Cell Culture}

HeLa cells were maintained in Dulbecco's modified Eagle's medium (DMEM; Gibco-BRL, Grand Island, N.Y., USA) supplemented with $10 \%$ fetal bovine serum (FBS; Biologica Industries, Kibbutz Beit Haemek, Israel), $50 \mathrm{U} / \mathrm{ml}$ of penicillin and $50 \mathrm{mg} / \mathrm{ml}$ streptomycin. Cells were incubated at $37^{\circ}$ with $5 \% \mathrm{CO}_{2}$ and separated for passage by $0.02 \%$ EDTA and $0.25 \%$ trypsin.

\section{Construction of CVB3-eGFP}

The strategy of CVB3 genome engineering is described in figure 1. The plasmid pMKS-1, containing the full-length genome of the CVB3 H3 strain [15], was kindly offered by Dr. J. Lindsay Whitton of the Scripps Research Institute (La Jolla, Calif., USA). A $S f i$ I restriction site and a $3 \mathrm{C}$ protease $\left(3 \mathrm{C}^{\text {pro }}\right)$ cleavage site of CVB3 were introduced immediately downstream of the initiation codon ATG of the viral ORF in pMKS-1 [16]. The $3 \mathrm{C}^{\text {pro }}$ cleavage site facilitates the detachment of the fusion protein coded by the inserted gene at the $S f i$ site from the CVB3 polyprotein and ensures the assembly and maturation of progeny virions. This strategy for the construction of the recombinant CVB3 virus has been used previously [16]. Briefly, pMKS1 was linearized by restriction enzyme $S f i$ I and the ends of linearized pMKS-1 were treated with calf intestinal alkaline phosphatase. egfp ORF (714 bp in length) was obtained by PCR amplification from the template of peGFPN1 (Clontech Laboratories, Mountain View, Calif., USA) with primers of ATAGGCCTGAGAGGCCGTGAGCAAGGGCGAGG AGCT (sense) and ATAGGCCTCTCTGGCCTTGTACAGCTCGTCCATGC (antisense; $S f i$ I sites are underlined). The $S f i$ I-treated pMKS-1 and egfp ORF were ligated at $4^{\circ}$ overnight (pMKS$1:$ egf $p=3: 1$ in quantity). $10 \mu \mathrm{l}$ of the ligation product, which was termed pMKS-1-eGFP, was used to transform competent $E$. coli $\mathrm{DH} 5 \alpha$. Three clones of pMKS-1-eGFP were selected and sequenced with the primer of AATACAGACATGGTGCGAAGAGT.

Virus Recovery and Plaque Assay

$1 \times 10^{5}$ HeLa cells were seeded in 24-well plates and maintained with $500 \mu$ l of DMEM medium with $10 \%$ FBS, but without antibiotics, for $24 \mathrm{~h}$ (about $70 \%$ confluent). $0.8 \mu \mathrm{g}$ pMKS-1-eGFP and $2 \mu \mathrm{l}$ of Lipofectamine 2000 (Invitrogen, Carlsbad, Calif., USA) were diluted with $100 \mu$ l of OPTI-MEM I medium, respectively. Then the diluted pMKS-1-eGFP and Lipofectamine 2000 were mixed and incubated for $20 \mathrm{~min}$. The mixture was applied to transfect HeLa cells. 2 wells of HeLa cells were transfected with peGFP-N1 and used as positive control, while another 2 wells of HeLa cells were transfected with Lipofectamine 2000 only and used as negative control. Cytopathic effects and eGFP expression in the transfected cells were checked starting $24 \mathrm{~h}$ after transfection. The cells were frozen and thawed 3 times when green fluorescence could be viewed. The recovered virus in the cell lysates was designated as CVB3-eGFP.

To purify the recovered viruses and estimate their virulence, 10 -fold serial dilutions of the harvested cell lysate were prepared in DMEM. HeLa cells cultured in 6-well plates $\left(2 \times 10^{5}\right.$ cells/well $)$ were infected with $450 \mu \mathrm{l}$ of each serial dilution. The plates were incubated at $37^{\circ}$ for $1 \mathrm{~h}$. After the incubation, the cells were overlaid with $2 \mathrm{ml}$ of DMEM containing 5\% FBS and 1.6\% agarose, and incubated at $37^{\circ}$ for $72 \mathrm{~h}$ in the dark. The cells were then stained with $0.05 \%$ neutral red for $1 \mathrm{~h}$. The plaque-forming units 

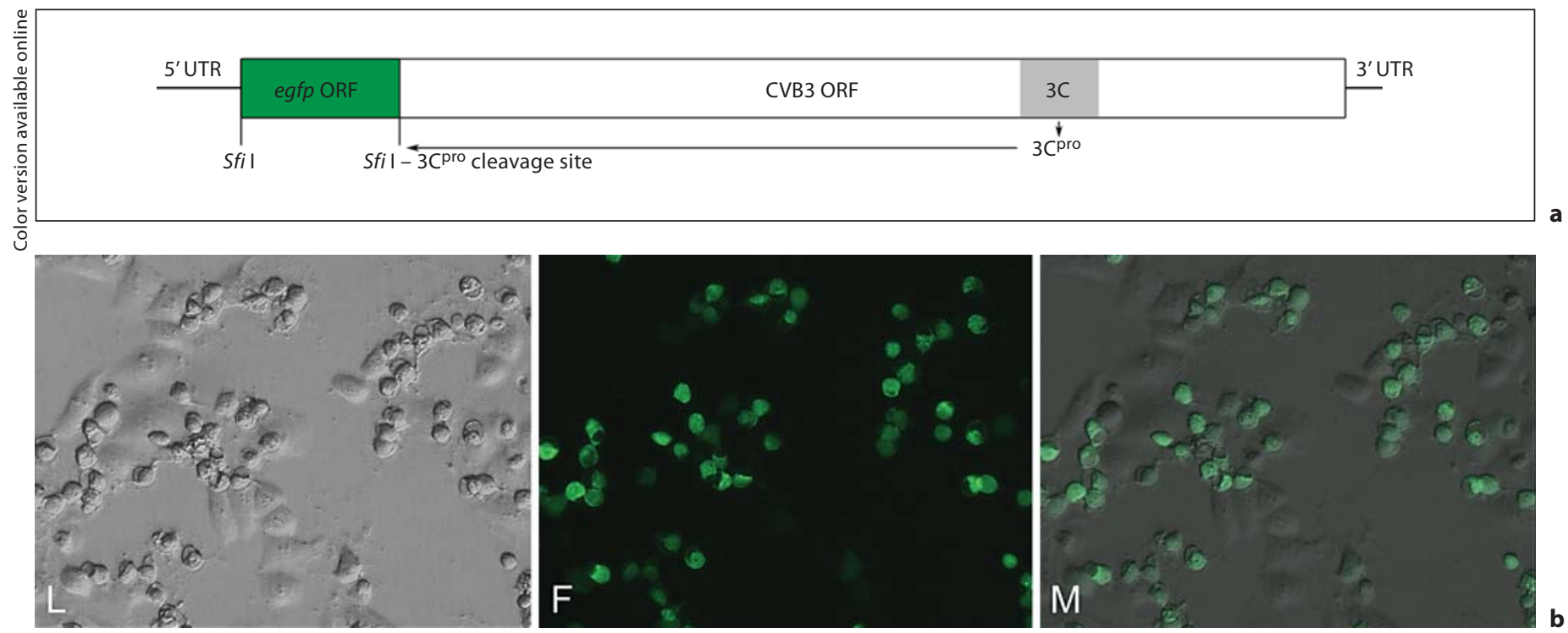

Fig. 1. The construction of CVB3-eGFP. a Strategy for the construction of CVB3-eGFP. A $S f i$ I restriction site followed by the $3 C^{\text {pro }}$ cleavage site was inserted immediately downstream of the initiation codon (ATG) of the ORF of CVB3 genome. egfp ORF was inserted into the $S f i$ I site. eGFP was expected to be detached from the viral polyprotein by the cleavage of viral $3 \mathrm{C}^{\text {pro }}$. b eGFP expression in HeLa cells infected with CVB3-eGFP for $24 \mathrm{~h}$ (×200). L = Light microscope; $\mathrm{F}=$ fluorescent microscope with 488-nm excitation; $\mathrm{M}=$ the merged image of $\mathrm{L}$ and $\mathrm{F}$. were determined as described previously [18]. 3 plaques of each recovered virus were applied to amplification and sequencing. The virus purified through plaque was defined as the first generation of CVB3-eGFP progenies.

\section{Sequencing}

CVB3-eGFP was serially passaged in HeLa cells, and total RNA from each of the 5 passages (from the 2 nd to the 6 th passage) was extracted with TRIzol Reagent (Invitrogen). $1 \mu$ l of the extracted RNA was applied to RT-PCR to amplify the viral sequence among which the egfp ORF was inserted. The RT-PCR product was then applied to $1.5 \%$ agarose gel electrophoresis. The primers for RTPCR amplification were GGCGGCAGTGTGTCGTAACGGGCAAC (P1, sense) and GCGTGGTTCTGTGAACTTGCCCGGG (P2, antisense; fig. 2). The amplified product is supposed to be $1,169 \mathrm{bp}$ in length if it contains the intact egfp gene, otherwise the egfp gene was deleted if the amplified product was $455 \mathrm{bp}$ in length (fig. 2a). The amplified products were cloned into pMD19-T simple vector (TaKaRa, Otsu, Japan) by the TA cloning method according to the manufacturer's instruction and then sequenced.

\section{Fluorescence Imaging}

The 6th passage of CVB3-eGFP cultured in HeLa cells was subjected to plaque assay as described previously [18]. All plaques were collected and inoculated to HeLa cells in 6-well plates, respectively. eGFP expression in the HeLa cells infected with these plaque-forming viruses was observed by fluorescent microscope with $488-n m$ excitation. The plates were then imaged by IVIS Lumina Imaging System (Xenogen, Alameda, Calif., USA) with 450to $490-\mathrm{nm}$ excitation and 10-second exposure.

\section{Results}

\section{Virus Construction and Purification}

Sequencing results showed that the sequence of egfp ORF and the flanking viral sequences in pMKS-1-eGFP were identical to that of the original design. At $48 \mathrm{~h}$ posttransfection, green fluorescence was observed in $\mathrm{HeLa}$ cells transfected with pMKS-1-eGFP. The transfected cells were frozen-thawed 3 times and subjected to plaque purification. The genomic sequence of the purified CVB3-eGFP was also confirmed by sequencing. Green fluorescence was clearly shown in HeLa cells infected with CVB3-eGFP purified through plaque (fig. 1). The virulence of CVB3-eGFP was as high as $1.17 \times 10^{8}$ plaqueforming units/ml, though this titer was about $1 \log$ unit lower than that of CVB3 without the insertion of egfp.

\section{Destabilization of the CVB3-eGFP Genome}

Genomic deletions of CVB3-eGFP were continuously observed from the 2nd passage of CVB3-eGFP cultured in HeLa cells, as demonstrated by RT-PCR amplification of egfp ORF and the flanking viral sequences (fig. 2a). RT-PCR showed that 3 fragments were consistently able to be amplified in the progenies of CVB3-eGFP from the 2 nd passage to the 6 th passage (fig. $2 \mathrm{~b}$ ). This result sug- 
Fig. 2. The genomic stability of CVB3eGFP passaged in HeLa cells. a The strategy of RT-PCR primer design for the evaluation of the genomic stability of CVB3eGFP. The RT-PCR product is expected to be 1,169 bp in length if there is an integrated egfp ORF in the genome of CVB3-eGFP. Otherwise, the egfp ORF is deleted if the RT-PCR product is $455 \mathrm{bp}$ in length. $\mathbf{b}$ The RT-PCR products of CVB3-eGFP serially passaged in HeLa cells for 2-6 times. Lane 1 is a DNA ladder (DL2000, TaKaRa). Lanes 2 and 3 are RT-PCR products using control template. In lane 4 , the RT-PCR template was replaced by water while other components of the amplification reaction remained the same as the one in lane 2 . Lanes 5-9 are RT-PCR products of CVB3eGFP from the 2 nd to the 6 th passage.
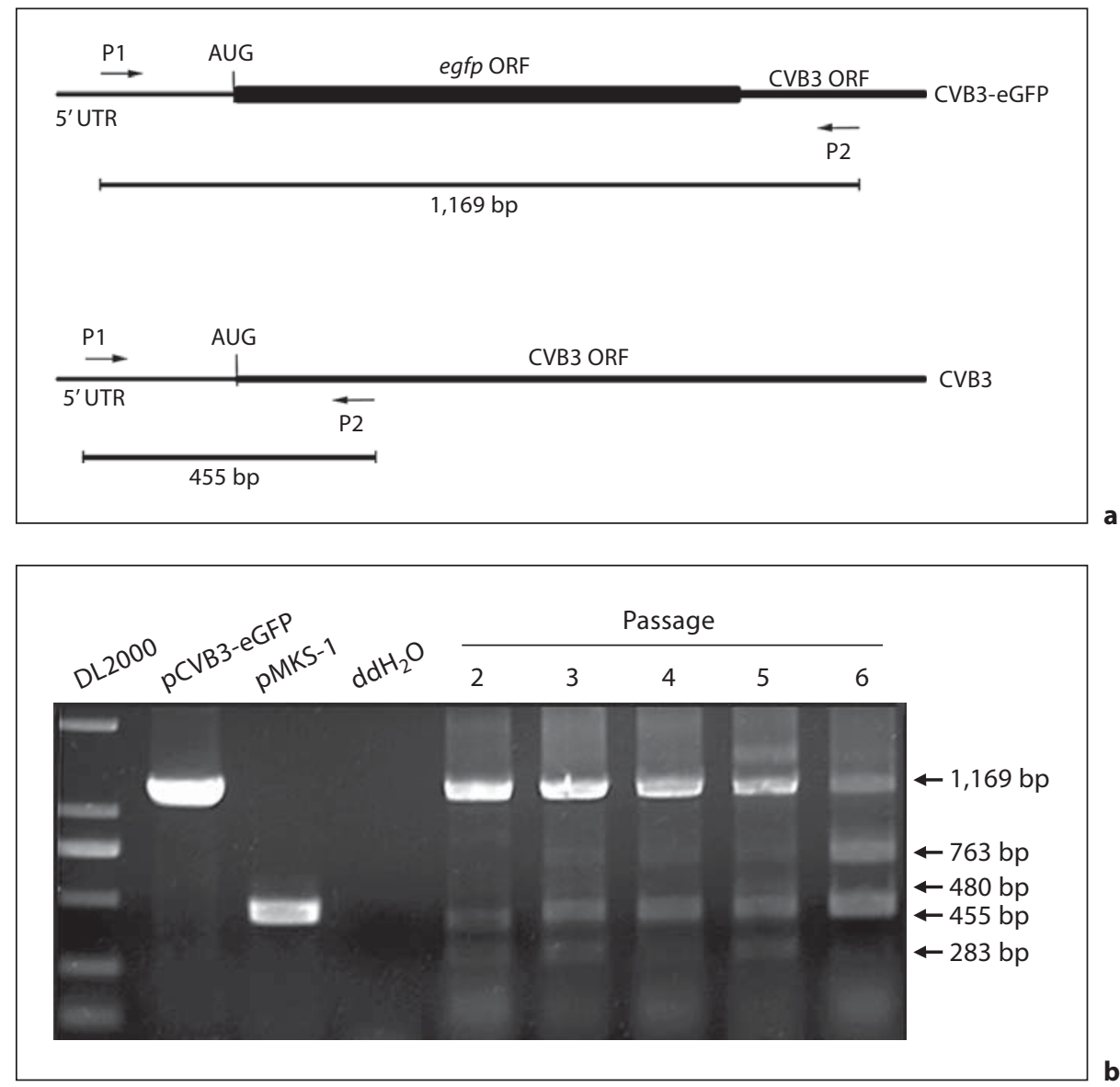

gests that genomic deletion occurred at certain preferred sites, but not randomly, in the genome of CVB3eGFP.

Each of the fragments obtained by RT-PCR was cloned into pMD19-T as described above. Three to six colonies from each clone were selected for sequencing. The sequencing results showed that the length of the 3 fragments was 763, 480 and $283 \mathrm{nt}$, respectively. The envisaged fragment of $450 \mathrm{nt}$ corresponding to the exact deletion of egfp from the CVB3-eGFP genome was not found in the amplified products. Alignment with the sequence of CVB3-eGFP prior to the passage of the progenies showed that the fragment of $763 \mathrm{nt}(\Delta 744-1,150)$ was the result of losing part of the $5^{\prime}$ end of egfp ORF. The fragment of $480 \mathrm{nt}(\Delta 987-1,653)$ was the result of losing the 3 ' end of egfp ORF and part of the VP4 coding sequence of CVB3. The fragment of $283 \mathrm{nt}(\Delta 766-1,653)$ resulted from losing most of the $3^{\prime}$ end of egfp ORF and part of VP4 coding sequence (fig. 3a). Sequence alignment also showed that all of the 3 deletion patterns led to the termination of protein synthesis of viruses due to the loss of initiation codon ATG $(\Delta 744-1,150)$ or the shift of the coding frame $(\Delta 987-1,653, \Delta 766-1,653$; fig. 3b).

\section{eGFP Expression of CVB3-eGFP Progenies}

Based on the sequence alignment (fig. 3), all of the 3 types of deletions in the genome of CVB3-eGFP resulted in lethal mutants. However, it is not likely that the RTPCR primers used in this study could cover all types of deletions in the progenies of CVB3-eGFP. Therefore, it was possible that there were other deletions in addition to those revealed above. Could these ignored deletions have produced viable mutants? To answer this question, the 6th passage of CVB3-eGFP was examined by virus plaque assay. Before neutral red staining, the cells were screened with fluorescent microscope with 488-nm excitation. We found that all plaque-like cytopathic foci showed green fluorescence (fig. 4a). This result indicated that all the viable viruses expressed eGFP. After neutral 

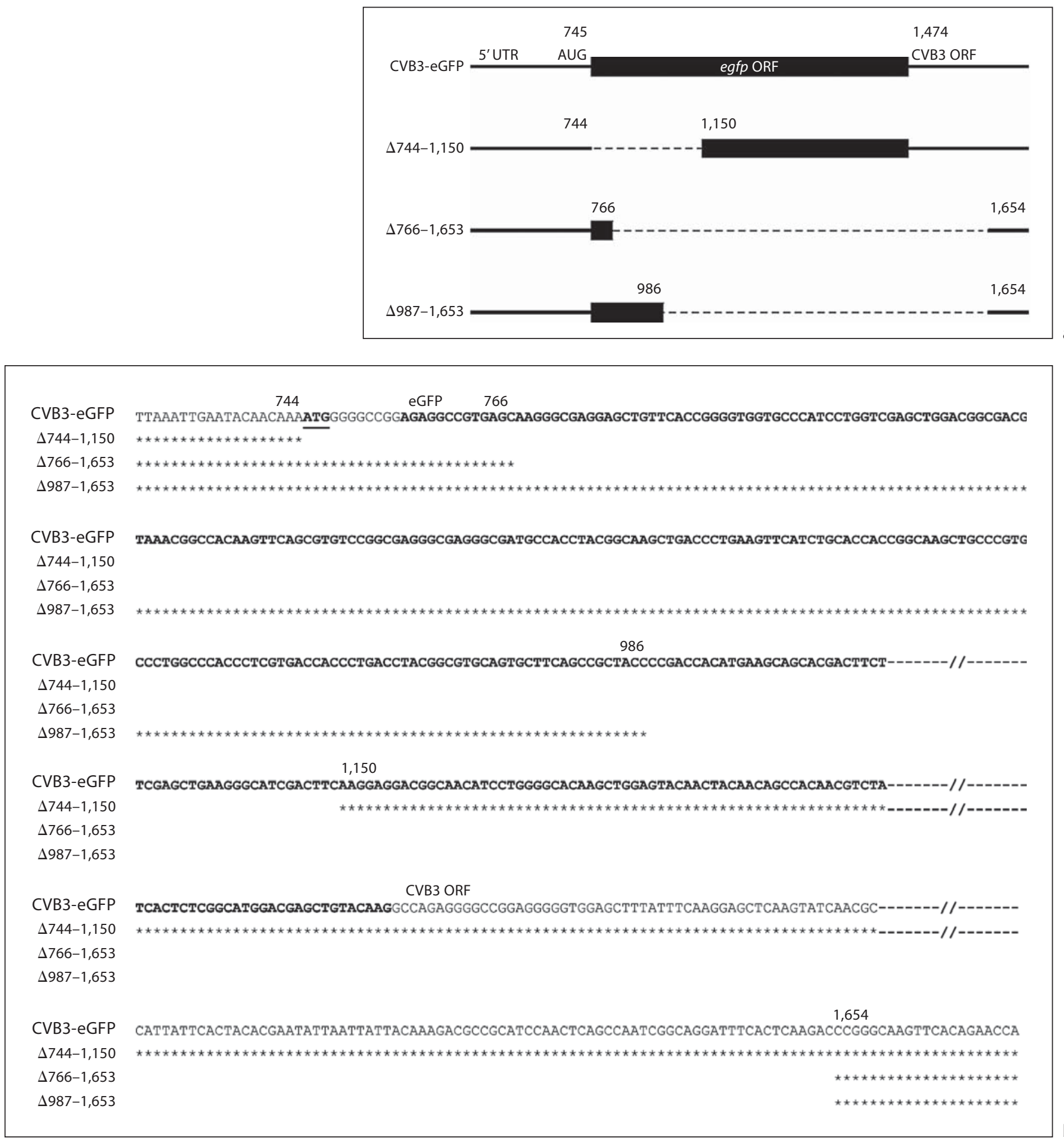

Fig. 3. Sequence alignment of the egfp ORF and adjacent sequences of CVB3-eGFP, with the RT-PCR products of $\Delta 744-1,150$, $\Delta 987-1,653$ and $\Delta 766-1,653$. CVB3-eGFP was passaged in HeLa cells continuously. Total RNAs of the 6th passage of CVB3-eGFP were reverse transcripted and amplified for the viral sequence including egfp ORF. The amplified products were cloned in pMD19$\mathrm{T}$ vector and sequenced. Sequencing of 3-6 colonies of each clone showed that $\Delta 744-1,150$ lost part of the $5^{\prime}$ end of egfp ORF. $\Delta 987-$ 1,653 lost the $3^{\prime}$ end of egfp ORF and part of the VP4 coding sequence of CVB3. a $\Delta 766-1,653$ lost most of the $3^{\prime}$ end of egfp ORF and part of the VP4 coding sequence. $\mathbf{b}$ All 3 deletion patterns led to the termination of protein synthesis due to either the deletion of initiation codon ATG $(\Delta 744-1,150)$ or the coding frame shifts ( $\Delta 987-1,653, \Delta 766-1,653)$. 
a

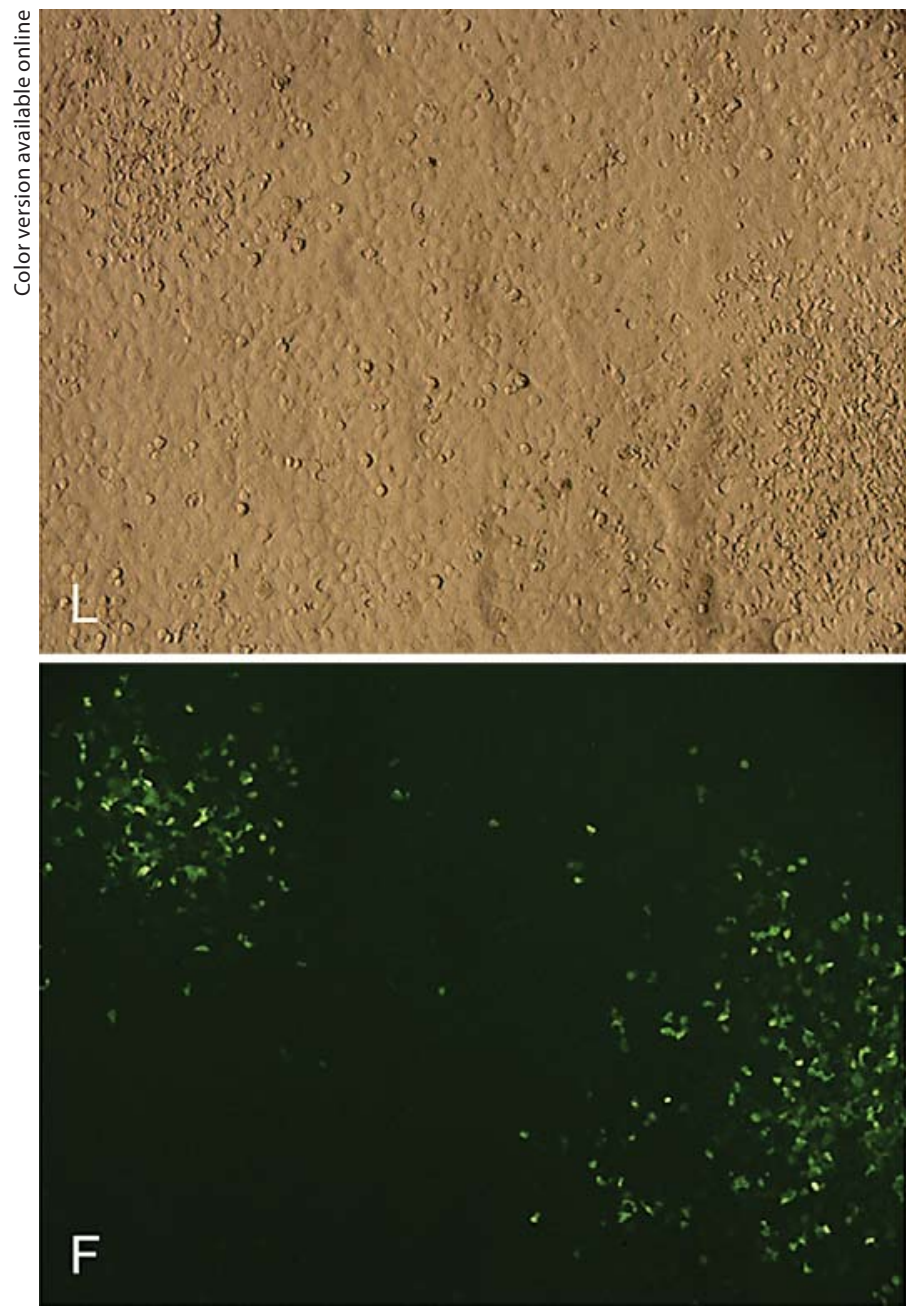

Fig. 4. The fluorescence of the plaques of the 6th passage of CVB3eGFP. a Before neutral red staining, the cells were viewed under fluorescent microscope with 488-nm excitation. All plaque-like cytopathic foci showed green fluorescence. $\mathrm{L}=$ Light microscopy; $\mathrm{F}=$ fluorescent microscopy. $\mathbf{b} 40$ plaques were observed in the HeLa cells after neutral red staining. All plaques were collected and applied to infect HeLa cells in 6-well plates. After 24-hour

red staining, 40 plaques were observed on the monolayer of HeLa cells. All of the plaques were picked up and applied to infect HeLa cells in 6-well plates. The infected HeLa cells were then cultured for $24 \mathrm{~h}$. Green fluorescence could be observed in all of the infected cells under fluorescent microscopy. Figure $4 \mathrm{~b}$ is an example of the results showed in 1 plate recorded by IVIS Lumina Imaging System with 450- to 490-nm excitation. The top 2 wells were uninfected HeLa cell controls, and the lower 4 wells were HeLa cells infected with plaque-forming viruses.

Destabilization of CVB3 Genome Fused with $e g f p$ b

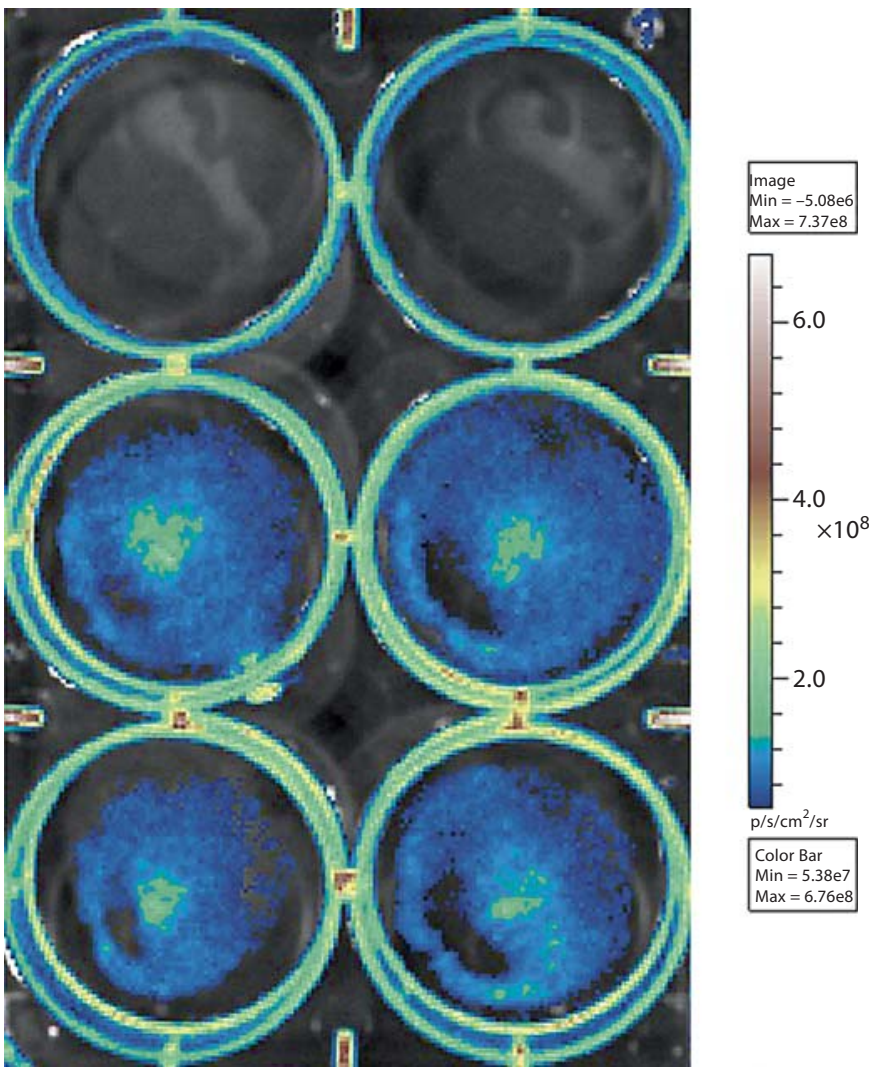

culture, the plates were detected by IVIS Lumina Imaging System (Xenogen) with 450- to 490-nm excitation and 10-second exposure. The top 2 wells were HeLa cell controls with viral infection, the lower 4 wells were HeLa cells infected with plaque-forming viruses. The autofluorescence of the plate could also be observed along the plastic walls.

\section{Discussion}

Group B coxsackieviruses are the established pathogens of severe human diseases including viral myocarditis and dilated cardiomyopathy. The genome of CVB is a single-stranded RNA with positive polarity, and contains only 1 ORF which encodes its polyprotein precursor. The polyprotein precursor is cleaved into the mature viral structural and functional proteins by virus-coded proteases. Unlike cellular mRNA, the 5' UTR of CVB directs the ribosomal entry and initiates the pro-

Intervirology 2011;54:268-275 
tein synthesis without poly(A)-binding protein dependence.

As a gene expression reporter, GFP has been used to monitor the replication and translation of the CVB genome in several studies [13-14]. Previously, it was reported that polioviruses fused with the ORF of $g f p$ or the GAG gene ( $\mathrm{gag}$ ) of human immunodeficiency virus type 1 showed severely impaired viral replication and was genetically unstable; the inserted sequences were rapidly deleted as early as the first growth cycle in HeLa cells [17]. This observation implies a possibility that a mutant mixture (quasispecies) could be produced in the viral pool during the replication of picornavirus with $g f p$, and consequently, the expression of $g f p$ may not be consistent to the viral gene expression. In this case, the role of $g f p$ as a reporter of viral gene expression would be compromised.

In this study, we constructed a CVB3 variant integrated with egfp ORF in the $5^{\prime}$ end of the CVB3 ORF. As it was expected, eGFP expression was observed in HeLa cells infected with CVB3-eGFP (fig. 1). To evaluate the stability of the CVB3-eGFP genome, purified CVB3eGFP was passaged continually in HeLa cells. RT-PCR amplification of the viral genomes with primers located at both ends of egfp ORF demonstrated that the genomic deletion of CVB3-eGFP began as early as the 2 nd passage (fig. 2b). This finding confirms that the insertion of the egfp gene can cause instability of the CVB3 genome.

RT-PCR showed that 3 deletion patterns could be consistently detected from the 2 nd to the 6 th passage (fig. $2 b$ ). These results suggest that genomic deletions are not random events, but occur at certain preferred sites of the CVB3-eGFP genome.

The RT-PCR products were cloned by the TA cloning method, and thereafter 3-6 clones of each fragment were sequenced. Sequence analysis revealed that the size of the RT-PCR products was 763,480 and $283 \mathrm{nt}$, respectively (fig. 3a). The 763-nt fragment lost sequence from the 744 th to 1,150 th nucleotide $(\Delta 744-1,150)$, the 480 -nt fragment lost sequence from the 987 th to 1,653 rd nucleotide $(\Delta 987-1,653)$ and the 283 -nt fragment lost sequence from the 766 th to 1,653 rd nucleotide $(\Delta 766-1,653)$. Sequence alignment showed that the initial codon AUG was removed in the $\Delta 744-1,150$ mutant. The reading frame shifts of the viral ORF happened in other mutants $(\Delta 987-$ 1,653 and $\Delta 766-1,653$; fig. 3 b). In $\Delta 987-1,653$ and $\Delta 766-$ 1,653 mutants, not only was most of the egfp ORF deleted, but part of the viral VP4 region was deleted as well. The 3 deletions led to a consequence of CVB3-eGFP protein synthesis termination, and theoretically were not likely to produce viable progeny.
Though all the genomic deletions mentioned above ended up with lethal mutations, there might be other deletions that were beyond the coverage of the primers we used. These possible deletions might also fail to generate viable mutants because more viral sequences would be removed. To verify this presumption, plaque assay was performed to isolate the progenies in the 6th passage of CVB3-eGFP. eGFP expression in the plaques was checked before and after neutral red staining. The results showed that all plaque-like foci on the monolayer of infected HeLa cells showed green fluorescence before neutral red staining (fig. 4a). All the 40 visible plaques stained with neutral red were collected to infect HeLa cells cultured in 6-well plates. All of the infected cells showed green fluorescence (fig. 4b). We did not find any green fluorescencenegative plaque. This observation confirms our presumption, but differs from the study of poliovirus with the $g f p$ gene [17], in which the genomic deletion produced viable mutants, though the plaque-forming characteristics of the mutants changed significantly.

We constructed 2 other CVB3 variants fused with the Renilla luciferase gene or red fluorescent protein mCherry gene. Similar genomic deletions were also found in both variants (data not shown). Therefore, we speculated that the stability of the picornavirus genome is a result of long-term natural adaptation. Any artificial modification, e.g. introducing a reporter gene, can greatly destabilize the picornavirus genome. On the other hand, a technical feature shared among the engineered viruses mentioned above is that the insertions are all located at the $5^{\prime}$ end of the viral ORF. Can the viral genome be relatively stable if the reporter gene is introduced at the $3^{\prime}$ end or other positions of the viral ORF? This speculation may be worth further investigation. A previous study [19] inserted adenovirus 2 (Ad2) hexon L1 loop (222 bp) into an artificial $2 \mathrm{~A}^{\text {pro }}$ site located in the middle of CVB3 ORF. RT-PCR analysis showed the genome of the resultant progeny virus remained stable within 10 passages, although mild genomic deletions were also observed in the 8 th and 10th passages. However, since the size of the Ad2 hexon L1 loop is far smaller than that of egfp and other reporter genes, it is hard to predict the stability of the viral genome if the egfp gene is inserted at this location.

Fierce genetic variation is a hallmark of RNA viruses. The predominant reason for this phenomenon is the misincorporation of nucleotides due to the lack of proofreading function of RNA polymerase during viral RNA synthesis [20]. For picornavirus, genomic recombination also contributes to its genetic change. Recombination can 
occur between 2 replicating poliovirus genomes in vivo and in vitro by a mechanism of crossover between sibling RNA strands during minus-strand synthesis of the replication-competent viral parent [20, 21]. Mueller and Wimmer [17] found that there were short repeat sequences in the immediate vicinity around the deletions of the $g f p$ gene in poliovirus, and proposed that these repeat sequences might facilitate nonhomologous RNA recombination during minus-strand synthesis and cause the deletion of the inserted reporter gene. In the case of CVB3eGFP, we could not find such a repeat sequence around the deleted sites. Therefore, another mechanism may be involved. Johansen and Morrow [22] revealed that poliovirus genomes $70 \%$ or smaller were not efficiently encapsidated. This provides a possible direction for further study of natural selections of CVB3-eGFP mutants.

Taken together, the egfp expression can be used as an indicator of the biosynthesis of CVB3-eGFP in a short period of time. However, it is not appropriate to use it for a long-duration observation. Furthermore, it is always necessary to purify the stocking viral pool by plaque assay before using CVB3-eGFP. The results based on CVB3-eGFP, especially in vivo observation, should be interpreted with caution. Overall, the genomic instability of picornaviruses integrated with reporter genes should be taken into account when related experimental data are interpreted.

\section{Acknowledgements}

We thank Dr. J. Lindsay Whitton for the gift of the CVB3 H3 strain. We are grateful to Prof. Kirk Knowlton (Cardiology Division, Department of Medicine, Medical School, University of California, San Diego, Calif., USA) for his insightful discussions about some aspects of this work. X.Z., Y.C. and H.G. are 7-year program students of Harbin Medical University. This work was supported by a grant from the National Natural Science Foundation of China (No. 30872231) and a grant from the Department of Education of Heilongjiang Province (No. 11531077). This work was also supported by the Infection and Immunity Key Laboratory, Harbin Medical University, Heilongjiang Province, China.

\section{References}

1 Racaniello VR: Picornaviridae: the virus and their replication; in Knipe DM, Howley PM, Griffin DE, Lamb RA, Straus SE, Martin MA, Roizman B (eds): Fields Virology, ed 5. Philadelphia, Lippincott Williams \& Wilkins, 2007, pp 795-838.

-2 Gan W, LaCelle M, Rhoads RE: Functional characterization of the internal ribosome entry site of eIF4G mRNA. J Biol Chem 1998; 273:5006-5012.

- 3 Jang SK, Kräusslich HG, Nicklin MJ, Duke GM, Palmenberg AC, Wimmer E: A segment of the $5^{\prime}$ nontranslated region of encephalomyocarditis virus RNA directs internal entry of ribosomes during in vitro translation. J Virol 1988;62:2636-2643.

4 Knowlton KU: CVB infection and mechanisms of viral cardiomyopathy. Curr Top Microbiol Immunol 2008;323:315-335.

5 Whitton JL, Cornell CT, Feuer R: Host and virus determinants of picornavirus pathogenesis and tropism. Nat Rev Microbiol 2005;3:765-776.

6 Yajima T, Knowlton KU: Viral myocarditis: from the perspective of the virus. Circulation 2009;119:2615-2624.

7 Bowles NE, Richardson PJ, Olsen EG, Archard LC: Detection of coxsackie-B- virusspecific RNA sequences in myocardial biopsy samples from patients with myocarditis and dilated cardiomyopathy. Lancet 1986;1: $1120-1123$

-8 Bledsoe AW, Jackson CA, McPherson S, Morrow CD: Cytokine production in motor neurons by poliovirus replicon vector gene delivery. Nat Biotechnol 2000;18:964-969.

-9 Kung SH, Wang YC, Lin CH, Kuo RL, Liu WT: Rapid diagnosis and quantification of herpes simplex virus with a green fluorescent protein reporter system. J Virol Methods 2000;90:205-212.

10 Jackson CA, Messinger J, Peduzzi JD, Ansardi DC, Morrow CD: Enhanced functional recovery from spinal cord injury following intrathecal or intramuscular administration of poliovirus replicons encoding IL-10. Virology 2005;336:173-183.

11 Heim R, Prasher DC, Tsien RY: Wavelength mutations and posttranslational autoxidation of green fluorescent protein. Proc Natl Acad Sci USA 1994;91:12501-12504.

12 Cubitt AB, Heim R, Adams SR, Boyd AE, Gross LA, Tsien RY: Understanding, improving and using green fluorescent proteins. Trends Biochem Sci 1995;20:448-455.

13 Feuer R, Mena I, Pagarigan R, Slifka MK, Whitton JL: Cell cycle status affect coxsackievirus replication, persistence and reactivation in vitro. J Virol 2002;76:4430-4440.

14 Lim BK, Shin JO, Lee SC, Kim DK, Choi DJ, Choe SC, Knowlton KU, Jeon ES: Long-term cardiac gene expression using a coxsackieviral vector. J Mol Cell Cardiol 2005;38:745751.

15 Knowlton KU, Jeon ES, Berkley N, Wessely $\mathrm{R}$, Huber S: A mutation in the puff region of VP2 attenuates the myocarditic phenotype of an infectious cDNA of the Woodruff vari- ant of coxsackievirus B3. J Virol 1996;70: 7811-7818.

16 Slifka MK, Pagarigan R, Mena I, Feuer R, Whitton JL: Using recombinant coxsackievirus $\mathrm{B} 3$ to evaluate the induction and protective efficacy of CD8+ T cells during picornavirus infection. J Virol 2001;75:2377-2387.

17 Mueller S, Wimmer E: Expression of foreign proteins by poliovirus polyprotein fusion: analysis of genetic stability reveals rapid deletions and formation of cardiovirus like open reading frames. J Virol 1998;72:20-31.

18 Zhong Z, Li X, Zhao W, Tong L, Liu J, Wu S, Lin L, Zhang Z, Tian Y, Zhang F: Mutations at nucleotides 573 and 579 within 5'untranslated region augment the virulence of coxsackievirus B1. Virus Res 2008; 135 : 255-259.

19 Höfling K, Tracy S, Chapman N, Kim KS, Smith Leser J: Expression of an antigenic adenovirus epitope in a group B coxsackievirus. J Virol 2000;74:4570-4578.

20 Kirkegaard K, Baltimore D: The mechanism of RNA recombination in poliovirus. Cell 1986;47:433-443.

21 Duggal R, Cuconati A, Gromeier M, Wimmer E: Genetic recombination of poliovirus in a cell-free system. Proc Natl Acad Sci USA 1997;94:13786-13791.

22 Johansen LK, Morrow CD: Inherent instability of poliovirus genomes containing two internal ribosome entry site (IRES) elements supports a role for the IRES in encapsidation. J Virol 2000;74:8335-8342. 